\title{
Colchicine for Coronary Heart Disease: A Meta-Analysis of Randomized Controlled Trials
}

\author{
Chunfeng Liao, ${ }^{1^{*}}$ Ping Liu, ${ }^{2^{*}}$ Yun Zhou, ${ }^{3} \mathrm{Di} \mathrm{Lu},{ }^{4} \mathrm{Qi} \mathrm{Wu}^{5}$ \\ ${ }^{1}$ Department of Cardiology, The First Hospital Of Changsha, Hunan Province, China; ${ }^{2}$ Department of Respiratory and Critical Care \\ Medicine, The First Hospital Of Changsha, Hunan Province, China; ${ }^{3}$ Department of Orthopedics, The First Hospital Of Changsha, \\ Hunan Province, China; ${ }^{4}$ Department of Ultrasound, Dianjiang County Hospital of Traditional Chinese Medicine, Chongqing; \\ ${ }^{5}$ Department of Critical Care Medicine, The First Hospital Of Changsha, Hunan Province, China
}

\section{ABSTRACT}

Background: The efficacy of colchicine administration for coronary heart disease remains controversial. We conducted a systematic review and meta-analysis to explore the influence of colchicine administration versus placebo on treatment efficacy for coronary heart disease.

Methods: We have searched PubMed, Embase, Web of Science, EBSCO, and Cochrane Library databases through May 2021 for randomized controlled trials (RCTs) assessing the effect of colchicine administration versus placebo in patients with coronary heart disease. This meta-analysis was performed using the random-effects model.

Results: Six RCTs involving 6,321 patients were included in the meta-analysis. Overall, compared with control groups for coronary heart disease, colchicine intervention can significantly reduce major adverse cardiovascular events (odds ratio [OR] $0.74 ; 95 \%$ confidence interval $[\mathrm{CI}] 0.59$ to $0.92 ; P=$ .006), but revealed no obvious impact on mortality $(\mathrm{OR}=0.93$; $95 \% \mathrm{CI}=0.63$ to $1.36 ; P=.69)$, serious adverse events (OR $0.71 ; 95 \%$ CI 0.31 to $1.61 ; P=.41$ ), or restenosis (OR 1.02 ; $95 \%$ CI 0.63 to $1.64 ; P=.95)$.

Conclusions: Colchicine treatment may be effective to reduce major adverse cardiovascular events in patients with coronary heart disease.

\section{INTRODUCTION}

Myocardial infarction has become a leading cause of mortality and morbidity, and percutaneous coronary intervention (PCI) is widely accepted as the most effective treatment strategy [Keeley 2003; Yang 2018; Russo 2018; Guo 2018; Echouffo-Tcheugui 2018]. Cardiomyocytes may be injured by acute restoration of myocardial blood flow [Ottani 2018;

Received fanuary 13, 2021; accepted fune 4, 2021.

* Chunfeng Liao, and Ping Liu contribute equally.

Correspondence: Yun Zhou, Di Lu, and Qi Wu, NO.311 Yingpan Road, Kaifu District, Changsha City, Hunan Province, China;fax: 008608789342584. (e-mail: 25002387@qq.com,31919599@qq.com, and 595497645@qq.com).
Cao 2018]. Vascular injury during PCI results in rapid neutrophil recruitment and subsequent inflammatory cascade that is associated with endothelial dysfunction and microvascular obstruction [Shu 2007; Aggarwal 2003]. Inflammation during PCI may also increase the risk of myocardial injury and mortality [Novack 2012].

Colchicine may have protective effects on coronary heart disease by inhibiting neutrophil chemotaxis and activity in response to vascular injury, active interleukin-1 $\beta$ (IL-1 $\beta$ ) and neutrophil-platelet aggregates [Paschke 2013; Martinon 2006; Shah 2016]. A 2-dose regimen of colchicine (1.2 $\mathrm{mg}$ followed by $0.6 \mathrm{mg}$ administered over an hour) showed rapid anti-inflammatory effects [Terkeltaub 2010]. In 1 randomized controlled trial (RCT) involving 4,745 patients, low-dose colchicine $(0.5$ $\mathrm{mg}$ once daily) after PCI resulted in a significantly lower risk of ischemic cardiovascular events than placebo [Tardif 2019].

Several studies have explored the efficacy of colchicine in patients with coronary heart disease, but the results were conflicting [Tardif 2019; Shah 2020; Akodad 2017]. With accumulating evidence, we therefore performed a systematic review and meta-analysis of RCTs to investigate the efficacy of colchicine administration versus placebo for coronary heart disease.

\section{METHODS}

This is a meta-analysis of previously published studies, and thus ethical approval and patient consent were not needed. The meta-analysis were conducted in adherence to Preferred Reporting Items for Systematic Reviews and Meta-Analyses (PRISMA) [Moher 2009].

\section{Search Strategy and Study Selection}

Two investigators independently searched the following databases (inception to May 2021): PubMed, Embase, Web of Science, EBSCO, and Cochrane Library. The electronic search strategy was conducted using the following keywords: "colchicine" AND "myocardial infarction" OR "percutaneous coronary intervention." We also checked the reference lists of the screened full-text studies to identify other potentially eligible trials. The inclusive selection criteria were as follows: (1) patients were diagnosed with coronary heart 
disease; (2) intervention treatments were colchicine administration versus placebo; and (3) study design was RCT.

\section{Data Extraction and Outcome Measures}

We extracted the following information: author, number of patients, age, male patients, history of myocardial infarction and PCI, and detailed methods in each group. Data were extracted independently by 2 investigators, and discrepancies were resolved by consensus. We also contacted corresponding authors to obtain data when necessary.

The primary outcomes were mortality and major adverse cardiovascular events (defined as repeated revascularization, nonfatal myocardial infarction, and cardiac death). Secondary outcomes included restenosis and serious adverse events (including cardiovascular and noncardiovascular adverse events such as gastrointestinal event, infection, pneumonia, and septic shock).

\section{Quality Assessment in Individual Studies}

Methodological quality of the included studies was independently evaluated using the modified Jadad scale [Jadad 1996], which uses 3 items: randomization (0 to 2 points), blinding (0 to 2 points), and dropouts and withdrawals (0 to 1 point), for a maximum possible total of 5 points. An article with Jadad score $\leq 2$ is considered to be of low quality, and Jadad score $\geq 3$ suggests high quality [Kjaergard 2001].

\section{Statistical Analysis}

We estimated the odds ratio (OR) with $95 \%$ confidence intervals (CIs) for all dichotomous outcomes (mortality, major adverse cardiovascular events, restenosis and serious adverse events). The random-effects model was used regardless of heterogeneity. Heterogeneity was reported using the I2 statistic, and $\mathrm{I} 2>50 \%$ indicated significant heterogeneity [Higgins 2002]. Whenever significant heterogeneity occurred, we searched for potential sources of heterogeneity by omitting 1 study in turn for the metaanalysis or performing subgroup analysis. Publication bias was not evaluated because of the limited number of included studies $(<10)$. All statistical analyses were performed using Review Manager version 5.3 (The Cochrane Collaboration, Oxford, UK).

\section{RESULTS}

Literature Search, Study Characteristics, and Quality Assessment Figure 1 is the detailed flowchart of the search and selection results. Initially, 348 potentially relevant articles were identified, and 5 RCTs were included in the final meta-analysis [Tardif 2019; Shah 2020; Akodad 2017; Zarpelon 2016; O'Keefe 1992; Tong 2020].

The baseline characteristics of the eligible RCTs in the meta-analysis are summarized in Table 1 . The 6 studies were published between 1992 and 2020, and the total sample size was 6,321. Colchicine was administered at doses ranging from 0.5 to $2 \mathrm{mg}$ daily. Among the 6 studies, 5 reported mortality [Tardif 2019; Shah 2020; Zarpelon 2016; Tong 2020; Briguori 2009], 3 reported major adverse cardiovascular events [Tardif 2019; Shah 2020; Tong 2020], 2 reported serious adverse events [Tardif 2019; Shah 2020], and 2 reported restenosis [Shah 2020; O'Keefe 1992]. Jadad scores of the 6 included
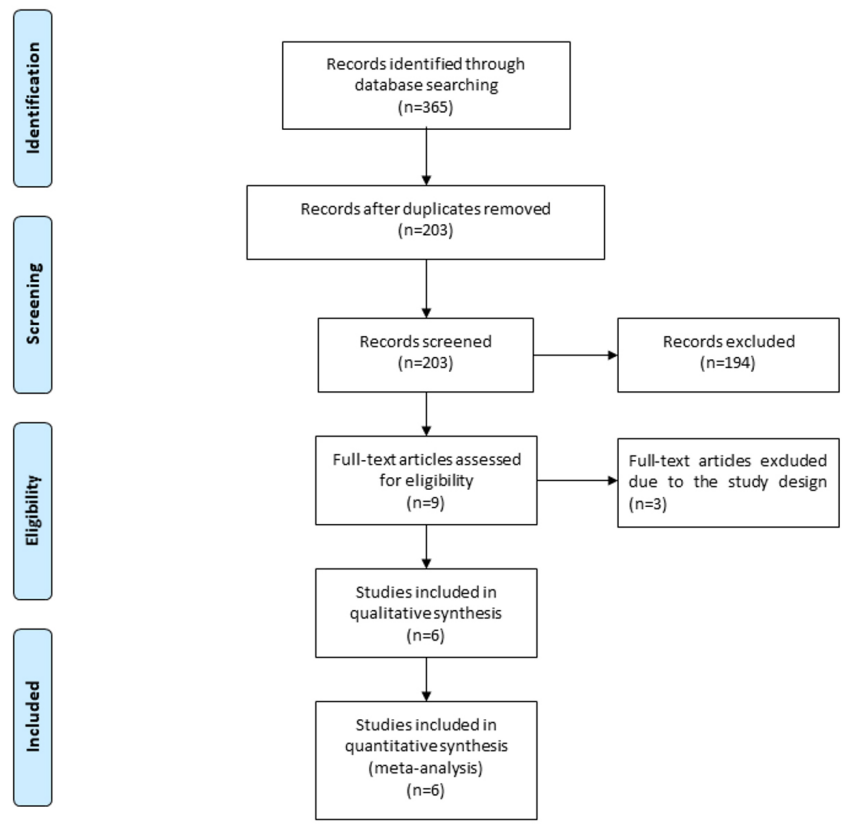

Figure 1. Flow diagram of study search and selection process.

Odds Ratio Odds Ratio

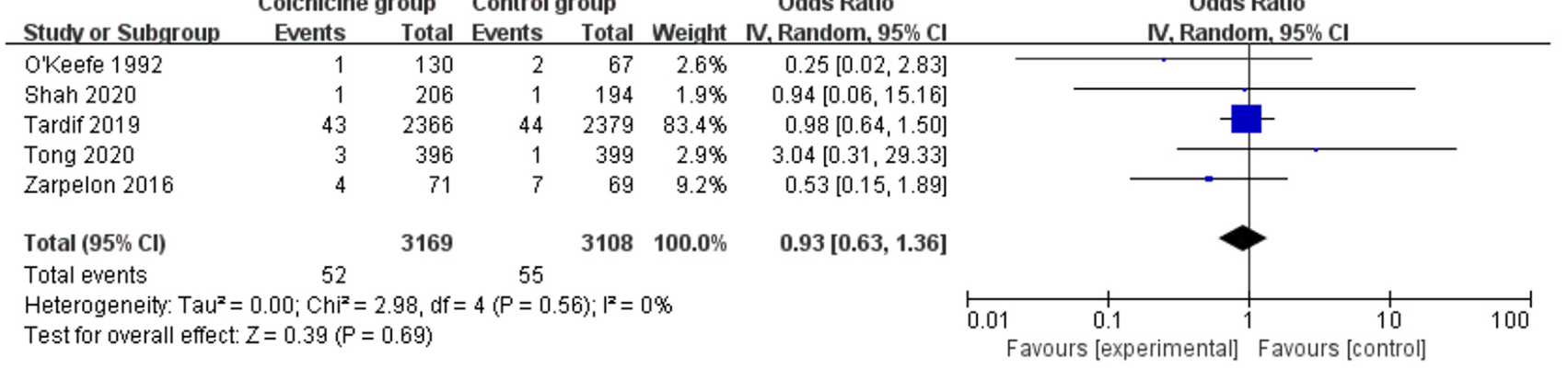

Figure 2. Forest plot for the meta-analysis of mortality. 
studies varied from 3 to 5 ; thus all 6 studies were considered to be of high quality.

\section{Primary Outcomes: Mortality and Major Adverse Cardio- vascular Events}

Outcome data were analyzed with the random-effects model, and the results suggest that compared with control groups for coronary heart disease, colchicine had no obvious impact on mortality (OR $0.93 ; 95 \%$ CI 0.63 to $1.36 ; P=$ .69 ), with no heterogeneity among the studies (I2 $=0 \%$, heterogeneity $P=.56$ ) (Fig. 2), but significantly reduced major adverse cardiovascular events (OR 0.74; $95 \%$ CI 0.59 to 0.92 ; $P=.006$ ), with low heterogeneity among the studies (I2 $=5 \%$, heterogeneity $P=.35$ ) (Fig. 3).

\section{Sensitivity Analysis}

Low or even no heterogeneity was observed among the included studies for the primary outcomes; thus we did not perform sensitivity analysis.

\section{Secondary Outcomes}

Compared with control groups for patients with coronary heart disease, colchicine revealed no obvious impact on serious adverse events (OR $0.71 ; 95 \%$ CI 0.31 to $1.61 ; P=.41)$ (Fig. 4) or restenosis (OR 1.02; $95 \%$ CI 0.63 to $1.64 ; P=.95$ ) (Fig. 5).

\section{DISCUSSION}

Anti-inflammatory therapy remains a promising option to reduce cardiovascular risk in patients with coronary heart disease. Preprocedural administration of high-intensity statin therapy has been documented to decrease myocardial injury and myocardial infarction in patients with acute coronary syndrome [Briguori 2009; Pasceri 2004; Patti 2007]. The anti-IL- $\beta$ antibody canakinumab was associated with reduced major adverse cardiovascular events by lowering IL-6 and high-sensitivity C-reactive protein concentrations in patients with prior myocardial infarction [Ridker 2017]. A rapid-acting

Table 1 Characteristics of included studies

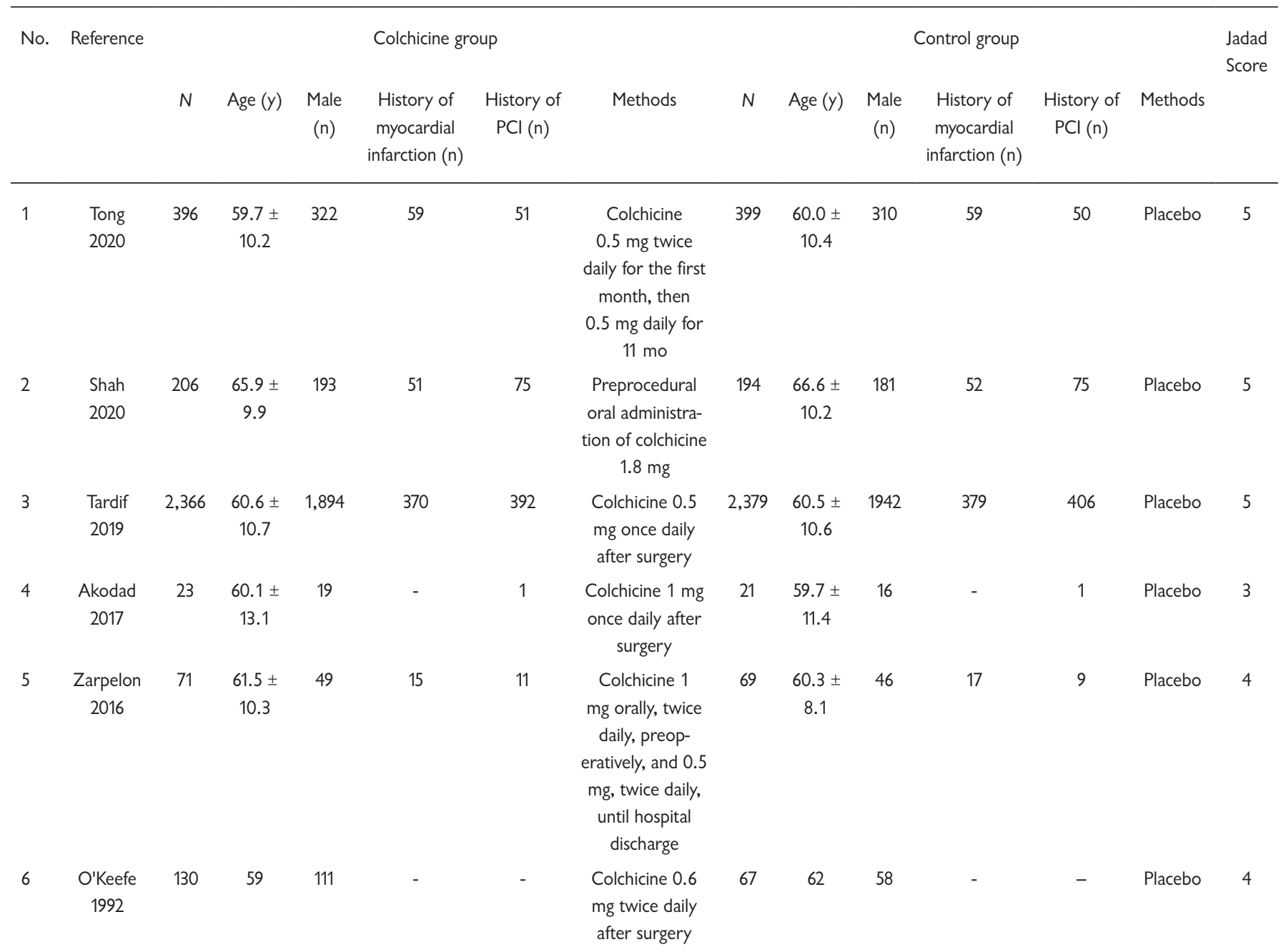




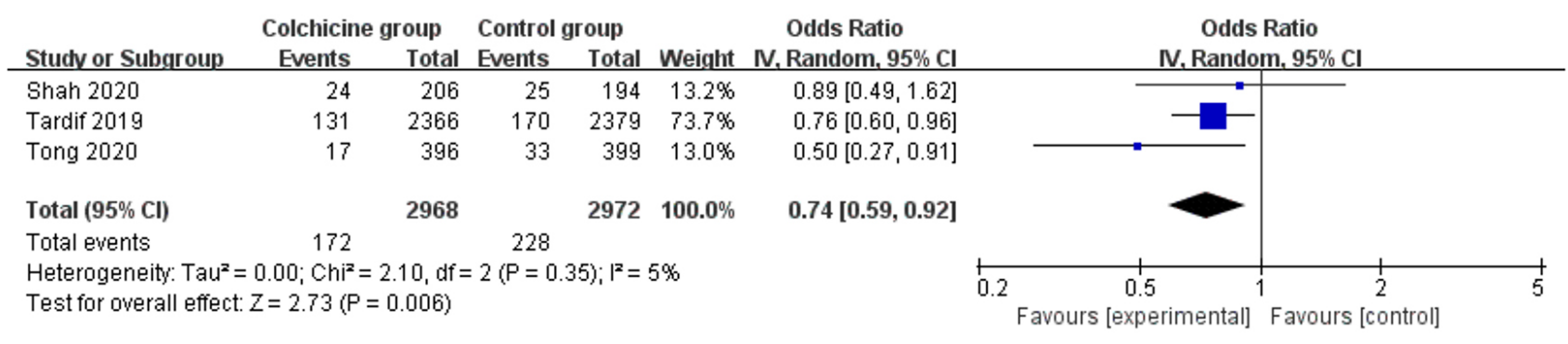

Figure 3. Forest plot for the meta-analysis of major adverse cardiovascular events.

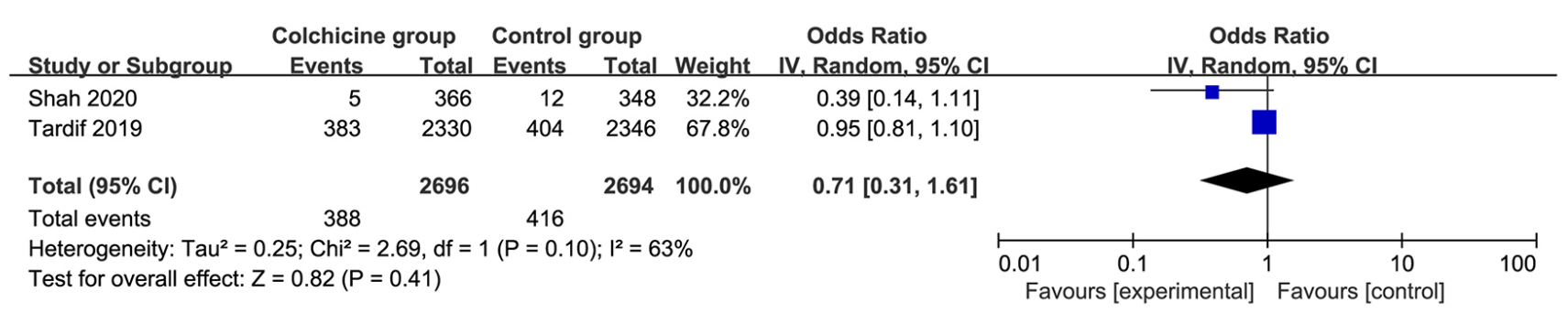

Figure 4. Forest plot for the meta-analysis of serious adverse events.

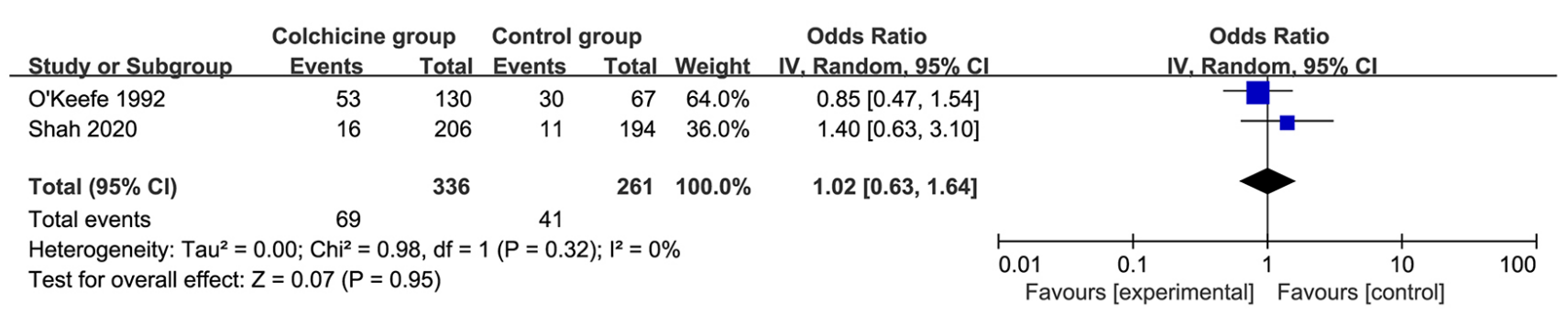

Figure 5. Forest plot for the meta-analysis of restenosis.

anti-inflammatory agent may be beneficial for patients with myocardial infarction [Borden 2011; Silva 2006].

Colchicine, an anti-inflammatory agent, was traditionally used to treat gout, suppress neutrophil homotypic adhesion, modulate neutrophil deformability, decrease neutrophil extravasation, suppress an enzymatic component of the inflammasome, and reduce IL-1 $\beta$ and IL-6 [Paschke 2013]. Decreased levels of neutrophil-platelet aggregates were observed after colchicine intervention and improved outcomes after PCI [Nidorf 2007]. Our meta-analysis suggests that colchicine can substantially reduce the major adverse cardiovascular events in patients with coronary heart disease but reveals no obvious influence on mortality, serious adverse events, or restenosis.

Although there was no significant heterogeneity in this meta-analysis, different doses and methods of colchicine administration may have produced some bias. Colchicine was administered at doses ranging from 0.5 to $2 \mathrm{mg}$ daily. Colchicine was found to decrease major adverse cardiovascular events for coronary heart disease, but there is no benefit of colchicine on mortality, which may be attributable to the low dose and only preoperative use of colchicine. In 1 RCT involving 140 patients with coronary heart disease, colchicine was used at a dose of $1 \mathrm{mg}$ orally, twice daily, preoperatively, and $0.5 \mathrm{mg}$, twice daily until hospital discharge. The results revealed that colchicine was associated with a lower death rate compared with placebo (5.6\% versus $10.1 \%$ ) [Zarpelon 2016]. These data indicate that high doses may provide improved benefits to reduce mortality.

This meta-analysis has several potential limitations. First, our analysis is based on only 6 RCTs, and more RCTs with large samples should be conducted to confirm these results. Next, the doses and methods of colchicine intervention were different, which may have an influence on the pooling results. Finally, the ideal methods of colchicine intervention remain elusive.

\section{Conclusions}

Colchicine intervention may reduce major adverse cardiovascular events in patients with coronary heart disease. 
REFERENCES

Aggarwal A, Schneider DJ, Terrien EF, Gilbert KE, Dauerman HL. Increase in interleukin-6 in the first hour after coronary stenting: An early marker of the inflammatory response. J Thrombosis Thrombolysis 2003;15:25-31.

Akodad M, Lattuca B, Nagot N, et al. COLIN trial: Value of colchicine in the treatment of patients with acute myocardial infarction and inflammatory response. Arch Cardiovasc Dis 2017;110:395-402.

Borden WB, Redberg RF, Mushlin AI, et al. Patterns and intensity of medical therapy in patients undergoing percutaneous coronary intervention. JAMA 2011;305:1882-1889.

Briguori C, Visconti G, Focaccio A, et al. Novel approaches for preventing or limiting events (Naples) II trial: impact of a single high loading dose of atorvastatin on periprocedural myocardial infarction. J Am Coll Cardiol 2009;54:2157-2163.

Cao B, Zhang C, Wang H, Xia M, Yang X. Renoprotective effect of remote ischemic postconditioning in patients with ST-elevation myocardial infarction undergoing primary percutaneous coronary intervention. Ther Clin Risk Manage 2018;14:369-375.

Echouffo-Tcheugui JB, Kolte D, Khera S, et al. Diabetes mellitus and cardiogenic shock complicating acute myocardial infarction. Am J Med 2018;131:778-786.

Guo L, Zhong L, Chen K, Wu J, Huang RC. Long-term clinical outcomes of optimal medical therapy vs. successful percutaneous coronary intervention for patients with coronary chronic total occlusions. Hellenic J Cardiol 2018;59:281-287.

Higgins JP, Thompson SG. Quantifying heterogeneity in a meta-analysis. Stat Med 21 2002;1539-1558.

Jadad AR, Moore RA, Carroll D, et al. Assessing the quality of reports of randomized clinical trials: Is blinding necessary? Controlled Clin Trials 1996;17:1-12.

Keeley EC, Boura JA, Grines CL. Primary angioplasty versus intravenous thrombolytic therapy for acute myocardial infarction: A quantitative review of 23 randomised trials. Lancet 2003;361:13-20.

Kjaergard LL, Villumsen J, Gluud C. Reported methodologic quality and discrepancies between large and small randomized trials in metaanalyses. Ann Intern Med 2001;135:982-989.

Martinon F, Pétrilli V, Mayor A, Tardivel A, Tschopp J. Gout-associated uric acid crystals activate the NALP3 inflammasome. Nature 2006;440:237-241.

Moher D, Liberati A, Tetzlaff J, Altman DG, PRISMA Group. Preferred reporting items for systematic reviews and meta-analyses: The PRISMA statement. J Clin Epidemiol 2009;62:1006-1012.

Nidorf M, Thompson PL. Effect of colchicine ( $0.5 \mathrm{mg}$ twice daily) on high-sensitivity C-reactive protein independent of aspirin and atorvastatin in patients with stable coronary artery disease. Am J Cardiol 2007;99:805-807.

Novack V, Pencina M, Cohen DJ, et al. Troponin criteria for myocardial infarction after percutaneous coronary intervention. Arch Intern Med 2012;172:502-508.

O'Keefe JH Jr, McCallister BD, Bateman TM, et al. Ineffectiveness of colchicine for the prevention of restenosis after coronary angioplasty. J Am Coll Cardiol 1992;19:1597-1600.

Ottani F, Limbruno U, Latini R, Misuraca L, Galvani M. Reperfusion in STEMI patients: Still a role for cardioprotection? Minerva Cardioangiol 2018;66:452-463.

Pasceri V, Patti G, Nusca A, et al. Randomized trial of atorvastatin for reduction of myocardial damage during coronary intervention: Results from the ARMYDA (Atorvastatin for Reduction of Myocardial Damage during Angioplasty) study. Circulation 2004;110:674-678.

Paschke S, Weidner AF, Paust T, et al. Technical advance: Inhibition of neutrophil chemotaxis by colchicine is modulated through viscoelastic properties of subcellular compartments. J Leukocyte Biol 2013;94:1091-1096.

Patti G, Pasceri V, Colonna G, et al. Atorvastatin pretreatment improves outcomes in patients with acute coronary syndromes undergoing early percutaneous coronary intervention: Results of the ARMYDA-ACS randomized trial. J Am Coll Cardiol 2007;49:1272-1278.

Ridker PM, Everett BM, Thuren T, et al. Antiinflammatory therapy with canakinumab for atherosclerotic disease. $\mathrm{N}$ Engl J Med 2017;377:1119-1131.

Russo JJ, Bagai A, Le May MR, Yan AT. Immediate non-culprit vessel percutaneous coronary intervention (PCI) in patients with acute myocardial infarction and cardiogenic shock: A swinging pendulum. J Thorac Dis 2018;10:661-666.

Shah B, Allen N, Harchandani B, et al. Effect of colchicine on plateletplatelet and platelet-leukocyte interactions: A pilot study in healthy subjects. Inflammation 2016;39:182-189.

Shah B, Pillinger M, Zhong H, et al. Effects of acute colchicine administration prior to percutaneous coronary intervention: COLCHICINEPCI randomized trial. Circul Cardiovasc Intervent 2020;13:e008717.

Shu J, Ren N, Du JB, et al. Increased levels of interleukin-6 and matrix metalloproteinase-9 are of cardiac origin in acute coronary syndrome. Scandinavian Cardiovasc J 2007;41:149-154.

Silva MA, Swanson AC, Gandhi PJ, Tataronis GR. Statin-related adverse events: A meta-analysis. Clin Ther 2006;28:26-35.

Tardif JC, Kouz S, Waters DD, et al. Efficacy and safety of low-dose colchicine after myocardial infarction. N Engl J Med 2019;381:2497-2505.

Terkeltaub RA, Furst DE, Bennett K, et al. High versus low dosing of oral colchicine for early acute gout flare: Twenty-four-hour outcome of the first multicenter, randomized, double-blind, placebo-controlled, parallel-group, dose-comparison colchicine study. Arthritis Rheumatism 2010;62:1060-1068.

Tong DC, Quinn S, Nasis A, et al. Colchicine in patients with acute coronary syndrome: The Australian COPS randomized clinical trial. Circulation 2020;142:1890-1900.

Yang HT, Xiu WJ, Zheng YY, et al. Invasive reperfusion after 12 hours of the symptom onset remains beneficial in patients with ST-segment elevation myocardial infarction: Evidence from a meta-analysis of published data. Cardiol J 2019;26:333-342.

Zarpelon CS, Netto MC, Jorge JC, et al. Colchicine to reduce atrial fibrillation in the postoperative period of myocardial revascularization. Arq Bras Cardiol 2016;107:4-9. 